\title{
The response of tropospheric circulation to perturbations in lower-stratospheric temperature
}

Article

Published Version

Haigh, J. D., Blackburn, M. and Day, R. (2005) The response of tropospheric circulation to perturbations in lower-

stratospheric temperature. Journal of Climate, 18 (17). pp. 3672-3685. ISSN 1520-0442 doi:

https://doi.org/10.1175/JCLI3472.1 Available at https://centaur.reading.ac.uk/110/

It is advisable to refer to the publisher's version if you intend to cite from the work. See Guidance on citing.

Published version at: http://dx.doi.org/10.1175/JCLI3472.1

To link to this article DOI: http://dx.doi.org/10.1175/JCLI3472.1

Publisher: American Meteorological Society

Publisher statement: (C) Copyright 2005 of the American Meteorological Society. The AMS Copyright Policy is available on the AMS web site at http://www.ametsoc.org

All outputs in CentAUR are protected by Intellectual Property Rights law, including copyright law. Copyright and IPR is retained by the creators or other copyright holders. Terms and conditions for use of this material are defined in the End User Agreement. 


\section{CentAUR}

Central Archive at the University of Reading

Reading's research outputs online 


\title{
The Response of Tropospheric Circulation to Perturbations in Lower-Stratospheric Temperature
}

\author{
JoAnna D. Haigh \\ Department of Physics, Imperial College, London, United Kingdom \\ Michael BLACKBURN \\ Centre for Global Atmospheric Modelling, University of Reading, Reading, United Kingdom \\ REBECCA DAY \\ Department of Physics, Imperial College, London, United Kingdom
}

(Manuscript received 14 April 2004, in final form 21 February 2005)

\begin{abstract}
A multiple regression analysis of the NCEP-NCAR reanalysis dataset shows a response to increased solar activity of a weakening and poleward shift of the subtropical jets. This signal is separable from other influences, such as those of El Niño-Southern Oscillation (ENSO) and the North Atlantic Oscillation (NAO), and is very similar to that seen in previous studies using global circulation models (GCMs) of the effects of an increase in solar spectral irradiance. The response to increased stratospheric (volcanic) aerosol is found in the data to be a weakening and equatorward shift of the jets.

The GCM studies of the solar influence also showed an impact on tropospheric mean meridional circulation with a weakening and expansion of the tropical Hadley cells and a poleward shift of the Ferrel cells. To understand the mechanisms whereby the changes in solar irradiance affect tropospheric winds and circulation, experiments have been carried out with a simplified global circulation model. The results show that generic heating of the lower stratosphere tends to weaken the subtropical jets and the tropospheric mean meridional circulations. The positions of the jets, and the extent of the Hadley cells, respond to the distribution of the stratospheric heating, with low-latitude heating forcing them to move poleward, and high-latitude or latitudinally uniform heating forcing them equatorward. The patterns of response are similar to those that are found to be a result of the solar or volcanic influences, respectively, in the data analysis.

This demonstrates that perturbations to the heat balance of the lower stratosphere, such as those brought about by solar or volcanic activity, can produce changes in the mean tropospheric circulation, even without any direct forcing below the tropopause.
\end{abstract}

\section{Introduction}

The role of the stratosphere in modulating the impact of solar variability on climate has been considered from a range of angles. From the point of view of radiative forcing of climate, it is clear that the increased stratospheric ozone concentrations that are present during periods of higher solar activity tend to reduce the direct (shortwave) radiative effect, but enhance the infrared component (Haigh 1994). However, the magnitude,

Corresponding author address: Joanna D. Haigh, Space and Atmospheric Physics, Blackett Laboratory, Imperial College, London SW7 2AZ, United Kingdom.

E-mail: j.haigh@imperial.ac.uk and even the sense of the net effect, is uncertain, with estimates of the amplification by solar-induced ozone of the net radiative forcing ranging from about $-25 \%$ (Wuebbles et al. 1998) to $+45 \%$ (Hansen et al. 1997; Larkin et al. 2000), and being largely dependent on assumptions concerning the distribution of the ozone increase. Narrowing these uncertainties is very important in terms of assessing the relative importance of solar radiative forcing in the context of other factors contributing to climate change; but, a consideration of radiative forcing alone does not provide a route to understand the atmospheric response, observed in various meteorological parameters, which is geographically nonuniform.

In the troposphere, these observations [e.g., studies 
of Northern Hemisphere (NH) upper-tropospheric thickness by van Loon and Shea (2000) or of zonal mean temperature by Haigh (2003)] tend to show the largest effects in the midlatitudes, suggesting a dynamical response to the solar forcing. One theory to explain these effects has been developed by Kodera and Kuroda (2002) who, using data analysis and numerical models, have shown that heating perturbations in the Northern Hemisphere winter stratosphere may modulate the upward propagation of planetary waves and that the interaction can move downward to lower levels. Other work (e.g., Shindell et al. 2001) has suggested that such wave propagation effects may shift the North Atlantic Oscillation (NAO) into a more positive phase. Gray et al. (2001) have shown a lagged correlation between equatorial upper-stratospheric winds and polar lower-stratospheric temperatures, again induced by modulating wave propagation. These ideas provide important routes to understand solar-climate interactions, but are effective only in the Northern Hemisphere winter. Other explanations must be found for the effects that occur throughout the year in both hemispheres.

In this paper we present an analysis of the observed response of zonal winds to solar, and other, influences and compare this with previously published studies using full global circulation models (GCMs). To aid in the interpretation of the observational and GCM results, we then investigate, using a simplified climate model, how generic heating to the lower stratosphere influences the circulation of the troposphere.

\section{Data analysis}

\section{a. The data and the methodology}

In a previous study (Haigh 2003) we presented a multiple linear regression analysis of zonal mean temperature data from the National Centers for Environmental Prediction-National Center for Atmospheric Research (NCEP-NCAR) reanalysis dataset, as provided by the Climate Diagnostics Center, in Boulder, Colorado (information available online at http://www.cdc.noaa. gov/). The multiple regression model (M. Allen 1998, personal communication) incorporated an autoregressive noise model of the order of one, and was used with the following 11 climate indices: a constant; a linear trend; predictors for solar activity [SOL; 10.7-cm flux from the National Geophysical Data Center (information online at ftp://ftp.ngdc.noaa.gov/STP/ SOLAR_DATA/)]; the quasi-biennial oscillation [QBO; zonal wind at $40 \mathrm{hPa}$ over Singapore (from B. Naujokat, Free University of Berlin)]; El NiñoSouthern Oscillation [ENSO; "cold tongue" index from the University of Washington at Seattle (information online at http://tao.atmos.washington.edu/data_sets/ cti/)]; stratospheric aerosol loading [VOL; presumed to be largely in response to volcanic eruptions, as constructed by Sato et al. (1993); a background level is assumed since 1996]; the NAO (from the University of East Anglia (online at http://www.cru.uea.ac.uk/ timo/ projpages/nao_update.htm)]; and four indices representing the amplitude and phase of both the annual and semiannual cycles.

At periods of maximum, relative to minimum, solar activity over the 11-yr solar cycle, regions of statistically significant (at the 95\% level) warming were detected in the low-latitude lower stratosphere and in vertical bands extending throughout the troposphere in the midlatitudes. The response to volcanic aerosol was found to be rather different, with a heating of the lower stratosphere occurring across all latitudes and a cooling of the troposphere, especially in the midlatitudes.

Here we apply the same technique to the NCEPNCAR reanalysis zonal mean zonal winds. The time period that is covered is January 1979-May 2002; earlier data are not used because of the well-known problem with the reanalysis in the stratosphere before the incorporation of satellite data in 1979 (see, e.g., Randel et al. 2000; Haigh 2003). All data and indices are monthly and (where appropriate) zonally averaged, and the analysis is carried out separately at each point on a grid of 73 latitudes and 17 isobaric levels.

A basic assumption of multivariate regression is that the indicators are independent and the effects are linearly additive. The cross correlations between the indices are shown in Table 1. This shows that the largest values occur between VOL and ENSO (0.326), and VOL and QBO (-0.106), so that the responses that are found for these indices may be mixed. The correlations between the solar index and other indices are small, less than 0.1 in all cases, so the scope for mixing these signals is less. Note, however, that the covariances between the indices are formally taken into account in the assessment of the statistical significance of the results presented in the next section.

\section{b. Results}

Figure 1a shows the zonal mean wind averaged over the inclusive period of January 1979-May 2002. The

TABLE 1. Cross correlations between the regression indices.

\begin{tabular}{lcccc}
\hline & SOL & QBO & ENSO & VOL \\
\hline NAO & 0.088 & -0.032 & 0.010 & 0.082 \\
VOL & -0.070 & -0.106 & 0.326 & \\
ENSO & -0.038 & -0.019 & & \\
QBO & -0.054 & & & \\
\hline
\end{tabular}



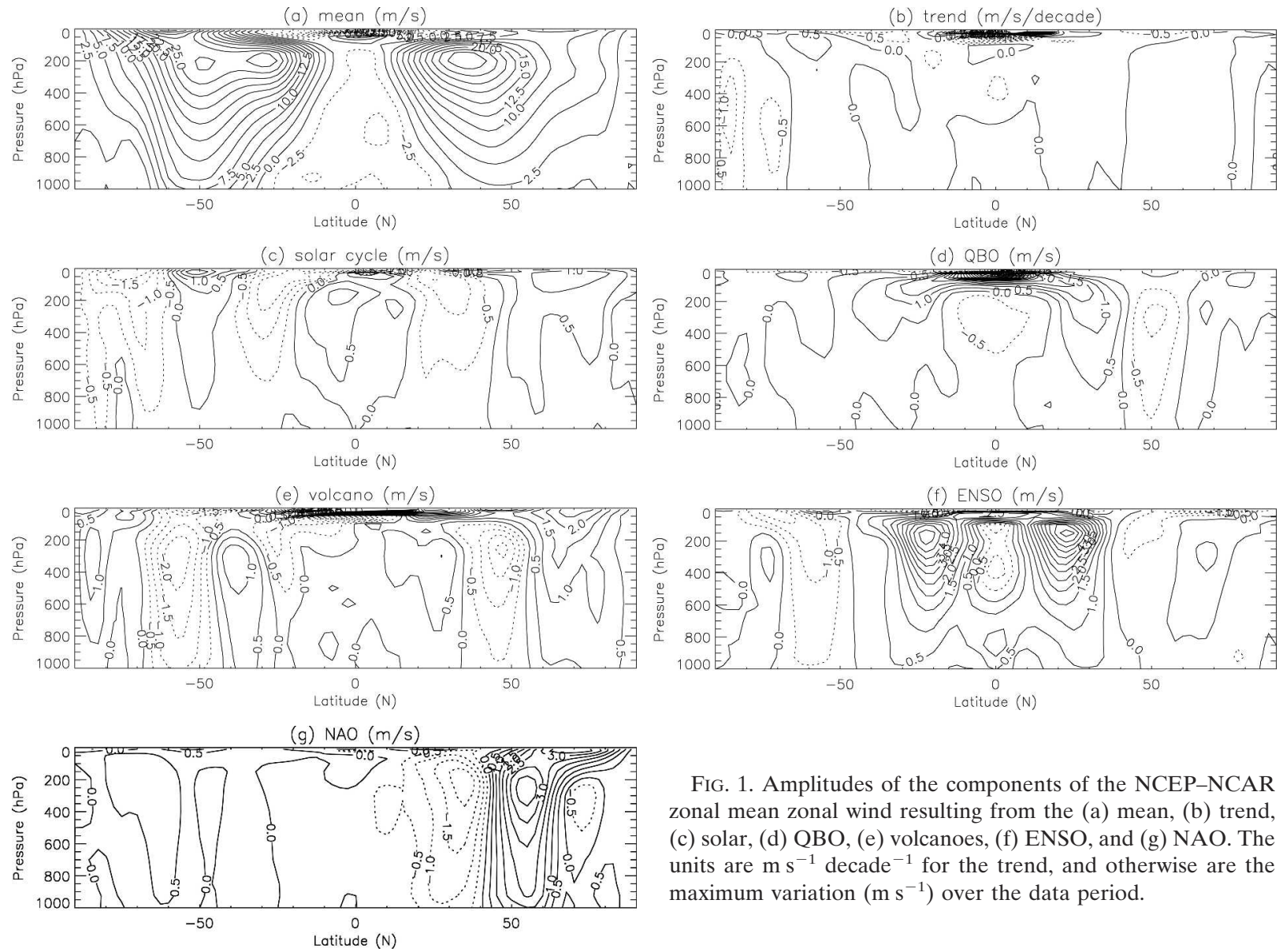

FIG. 1. Amplitudes of the components of the NCEP-NCAR zonal mean zonal wind resulting from the (a) mean, (b) trend, (c) solar, (d) QBO, (e) volcanoes, (f) ENSO, and (g) NAO. The units are $\mathrm{m} \mathrm{s}^{-1}$ decade $^{-1}$ for the trend, and otherwise are the maximum variation $\left(\mathrm{m} \mathrm{s}^{-1}\right)$ over the data period.

remaining panels show the components ascribed to the different indices as a function of latitude and pressure: (Fig. 1b) a linear trend, (Fig. 1c) the difference between minimum and maximum solar activity (which, over the period concerned, represents a typical 11-yr activity cycle), (Fig. 1d) a typical QBO cycle; (Fig. 1e) the difference between a period of low-stratospheric aerosol and that following the eruption of Mt. Pinatubo, and (Figs. 1f and 1g, respectively) the effect of large ENSO and NAO cycles. Statistical significance was estimated using a Student's $t$ test, taking into account the temporal autocorrelation of the data and any correlation between the indices. Details of this methodology can be found in standard statistical texts [we found that of von Storch and Zwiers (1999) to be particularly useful]. Significance values are not shown in the figure for reasons of clarity, but the regions of $>90 \%$ significance largely correspond to those that are shown for temperature by Haigh (2003), and generally occur where the signal is greater than about $0.5 \mathrm{~m} \mathrm{~s}^{-1}$. The lack of obvious noise in the figures, despite the regression having been carried out independently at each grid point, and the hemi-

spheric symmetry at low latitudes provide further reassurance of the reliability of the results.

The solar signal is seen to be composed of banded structures in the midlatitudes. The largest changes, a reduction of over $1 \mathrm{~m} \mathrm{~s}^{-1}$, are present on the equatorward sides of the jet cores, with positive values, of a few tenths of $1 \mathrm{~m} \mathrm{~s}^{-1}$, further poleward. Both the shape and the amplitude of this pattern are remarkably similar to those found in the GCM simulations of Haigh (1996, 1999 ) and, using an entirely different model, Larkin et al. (2000). These model studies included the effects of changes in solar spectral irradiance where the largest direct radiative impact, resulting from enhanced solar $\mathrm{UV}$, is found in the stratosphere.

The results clearly show a weakening and poleward shift of the jets when the sun is more active, again, as predicted by the model studies. The GCMs also predicted a response to higher levels of solar UV in the tropospheric mean meridional circulation. This consisted of a weakening and expansion of the Hadley cells and a poleward shift of the Ferrel cells. It is interesting to note that precisely these features, which are highly 
correlated with solar activity, have now been detected in NCEP-NCAR vertical velocity data (Gleisner and Thejll 2003).

The responses to the other indices show different patterns; Fig. 1e presents, for the volcanic aerosol, vertical bands at different latitudinal positions than those appearing in the solar case, which are also of a somewhat larger magnitude. As in the solar case, the jets are weaker, but for the volcanic aerosol case they appear to shrink in latitudinal extent, with a greater shift toward the equator. In the Northern Hemisphere, the NAO signal (Fig. 1g) is somewhat similar to that resulting from the sun, although it is shifted slightly equatorward. The temporal correlation between the SOL and NAO indices is 0.088 , so it is feasible that some of the NAO effect might be ascribed to the sun. The NAO signal in the Southern Hemisphere, however, is negligible, and the degree of hemispheric symmetry in the solar signal suggests that the result is robust; remember that the calculations are carried out independently at each grid point. Furthermore, the deduced solar signal is somewhat weaker in the Northern Hemisphere, so that arguments based on hemispheric symmetry would suggest that some of the solar signal might have been mapped onto the NAO, rather than vice versa. Some studies have suggested that solar activity may induce the NAO into a more positive phase (Shindell et al. 2001), which would mean that the two effects are not linearly independent, but also suggest that the solar signal that is deduced here would be a low estimate in the Northern Hemisphere.

\section{Investigations with a simplified global circulation model}

\section{a. The model and the experiments}

To understand the mechanisms underlying the observed tropospheric variability that are associated with solar and volcanic forcing, idealized forcing experiments have been performed using a simplified global circulation model (sGCM).

The model uses the spectral dynamical core, described by Hoskins and Simmons (1975), that is modified to include the angular momentum-conserving vertical discretization of Simmons and Burridge (1981), but retains the original sigma coordinate. All experiments use a T42L15 resolution, denoting triangular spectral truncation at a total wavenumber 42 , which is equivalent to an equatorial grid spacing that is close to $475 \mathrm{~km}$ for the equivalent linear transform grid; and 15 levels between the surface and $\sigma=0.0185$, with the level spacing chosen such that it provides particularly good resolution in the region of the tropopause [the model levels are at 18.5, 59.6, 106, 152, 197, 241, 287, $338,400,477,569,674,784,887$, and 967 in units of $\left.\sigma\left(1000^{-1}\right)\right]$.

The model's climate is maintained using the linear forcing and drag scheme of Held and Suarez (1994), in place of the moist parameterizations of a full GCM. Orography is omitted. Temperature is relaxed toward a zonally symmetric equilibrium distribution, on a time scale that is 40 days above $\sigma=0.7$ (representing radiation and deep, moist processes), but reduces to 4 days at the equatorial surface (representing the planetary boundary layer). Rayleigh friction is included below sigma $=0.7$, with a time scale of 1 day at the surface. Radiative forcing perturbations are represented by modifications of the equilibrium temperature distribution in the stratosphere.

The equilibrium temperature $T_{e}$ is a function only of latitude $\phi$, and pressure $p$ :

$$
\begin{aligned}
T_{e}(\phi, p)= & \max \left\{\left(T_{\text {tpeq }}-\Delta T_{\mathrm{tp}} \sin ^{2} \phi\right)\right. \\
& \times\left[T_{0}-\Delta T_{y} \sin ^{2} \phi-\left(\Delta \theta_{\mathrm{eq}} \cos ^{2} \phi\right.\right. \\
& \left.\left.\left.+\Delta \theta_{\mathrm{pl}} \sin ^{2} \phi\right) \log \left(\frac{p}{p_{0}}\right)\right]\left(\frac{p}{p_{0}}\right)^{\kappa}\right\},
\end{aligned}
$$

where $p_{0}$ is a reference surface pressure, $T_{\text {tpeq }}$ is the temperature of the equatorial tropopause, $\Delta T_{\mathrm{tp}}$ is the difference in temperature between the equatorial and polar tropopause, $T_{0}$ is the temperature of the surface at the equator, $\Delta T_{y}$ is the difference in surface temperature between equator and poles, and $\Delta \theta_{\mathrm{eq}}$ and $\Delta \theta_{\mathrm{pl}}$ are the lapse-rate factors (the increase in potential temperature with an increase in altitude of one pressure scale height) at the equator and poles, respectively. In all of the runs discussed here $p_{0}=1000 \mathrm{hPa}, T_{0}=315$ $\mathrm{K}, \Delta T_{y}=60 \mathrm{~K}, \Delta \theta_{\mathrm{eq}}=10 \mathrm{~K}$, and $\Delta \theta_{\mathrm{pl}}=0 \mathrm{~K}$. For the control run, $T_{\text {tpeq }}=200 \mathrm{~K}$ and $\Delta T_{\text {tp }}=0 \mathrm{~K}$.

The structure of the equilibrium temperature field and the resultant model temperature field for the control run are shown in Figs. $2 \mathrm{a}$ and $2 \mathrm{~b}$. The dynamical response to the equilibrium structure is such as to cause adiabatic warming at the mid- to high latitudes and cooling at low latitudes, so that although $T_{e}$ is uniform and constant above the tropopause, the model shows a positive latitudinal temperature gradient there, while in the troposphere the negative temperature gradients are reduced. The model's zonal wind structure is shown in Fig. 2c to aid in the assessment of the results of the model experiments discussed below.

Experiments with the model have been designed to investigate the effects of perturbations to the tempera- 
(a)

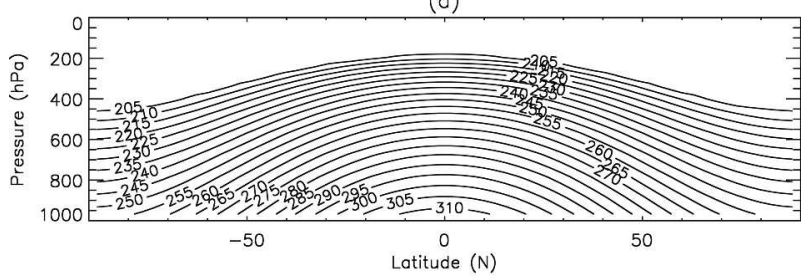

(b)

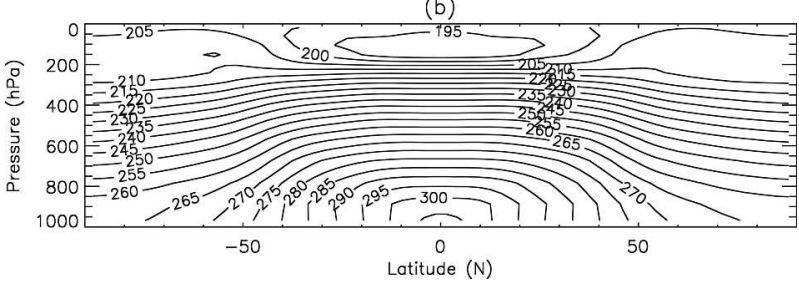

(c)

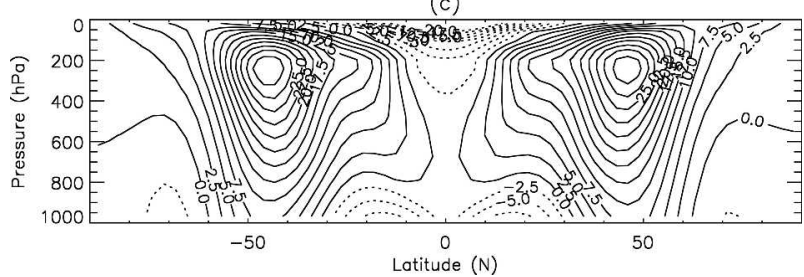

FIG. 2. Simple GCM fields: (a) the prescribed equilibrium temperature $T_{e}(\mathrm{~K})$; (b) the zonal mean temperature $(\mathrm{K})$ for the control run $\mathrm{C}$; and (c) the zonal mean zonal wind for run $\mathrm{C}$.

ture structure of the lower stratosphere, and to this effect comprise varying the values used for $T_{\text {tpeq }}$ and $\Delta T_{\mathrm{tp}}$. The values that are used are summarized in Table 2. Experiments U1 and U5 prescribe a uniform increase in $T_{e}$ of 1 and $5 \mathrm{~K}$, respectively, throughout the stratosphere. In experiments E1 and E5 the equatorial stratosphere is warmed by 1 and $5 \mathrm{~K}$, respectively, but this is gradually reduced to a zero increase at the poles. For experiment $\mathrm{P} 10$ the equatorial $T_{e}$ is unchanged, but the poles are warmed by $10 \mathrm{~K}$. Thus, we have representations of situations in which the whole stratosphere warms uniformly, or warms preferentially at low or high latitudes. The structure of these temperature changes is very simple, and the model runs are not intended to be direct simulations of solar (or volcanic)

TABLE 2. Summary of experiments with the simplified GCM.

\begin{tabular}{lcrll}
\hline \hline Run & $\begin{array}{c}T_{\text {tpeq }} \\
(\mathrm{K})\end{array}$ & $\begin{array}{c}\Delta T_{\text {tp }} \\
(\mathrm{K})\end{array}$ & $\begin{array}{c}\text { Length } \\
\text { (days) }\end{array}$ & $\begin{array}{c}\text { Increase in } \\
\text { stratospheric } \\
T_{e}(\mathrm{~K})\end{array}$ \\
\hline C & 200 & 0 & $2 \times 10000$ & None \\
U1 & 201 & 0 & $2 \times 10000$ & 1 \\
U5 & 205 & 0 & $2 \times 1000$ & 5 \\
E1 & 201 & 1 & $2 \times 10000$ & $\cos ^{2} \phi$ \\
E5 & 205 & 5 & $2 \times 1000$ & $5 \cos ^{2} \phi$ \\
P10 & 200 & -10 & $2 \times 1000$ & $10 \sin ^{2} \phi$ \\
\hline
\end{tabular}

effects. We shall see in section $3 c$, however, that the results may be useful in understanding the effects of perturbations to the real atmosphere, such as those outlined in section 2.

The $\mathrm{U}$ and $\mathrm{E}$ experiments were each carried out with the two different amplitudes, of 1 and $5 \mathrm{~K}$, in order to assess whether the patterns of response were independent of the magnitude of the forcing, and to what extent the response scaled with the forcing. The statistical significance of the responses is estimated independently at each grid point using a Student's $t$ test, taking into account the reduction in the number of degrees of freedom resulting from autocorrelations in the time series. The signals produced by the 10 - and $5-\mathrm{K}$ perturbations are much larger than the long-term variability in the fields, so that it was necessary to continue these runs for only 1000 days, after an initial spinup period of 200 days. For the 1-K experiments, and the control, runs of 10000 days were carried out. In all cases the effective number of data points has been doubled by using the results from the two hemispheres as independent simulations. This is possible because an analysis of the temporal correlations between equivalent points in the two hemispheres shows very low values (of a magnitude of $\sim 0.2$ ) across most of the hemisphere. ${ }^{1}$

\section{b. Results}

\section{1) Temperature}

The anomalies in zonal mean temperature for the perturbation experiments are shown in Fig. 3. All of the experiments show heating in the stratosphere, as would be expected; but in each case there are regions near the poles where the increase in stratospheric temperature is larger than the imposed change in $T_{e}$, resulting from the greater descent and resulting adiabatic warming. All of the experiments also show regions of statistically significant warming in the troposphere, despite there being no imposed perturbation to $T_{e}$ there. In experiments U5 and U1 there are vertical bands of cooling near $40^{\circ}$ latitude, with bands of warming either side. The pattern of the tropospheric response is very similar in experiment P10, but with somewhat larger magnitudes. The E experiments both show the same pattern, which is different to that produced by the $\mathrm{U}$ and $\mathrm{P}$ experiments; there is now a band of warming near $50^{\circ}$, flanked by cooling bands near $30^{\circ}$ and $65^{\circ}-70^{\circ}$.

\footnotetext{
${ }^{1}$ In fact, opposite points in the two hemispheres generally show a small anticorrelation, presumably resulting from small movements of mass across the equator, so that averaging them does not artificially enhance any signal.
} 

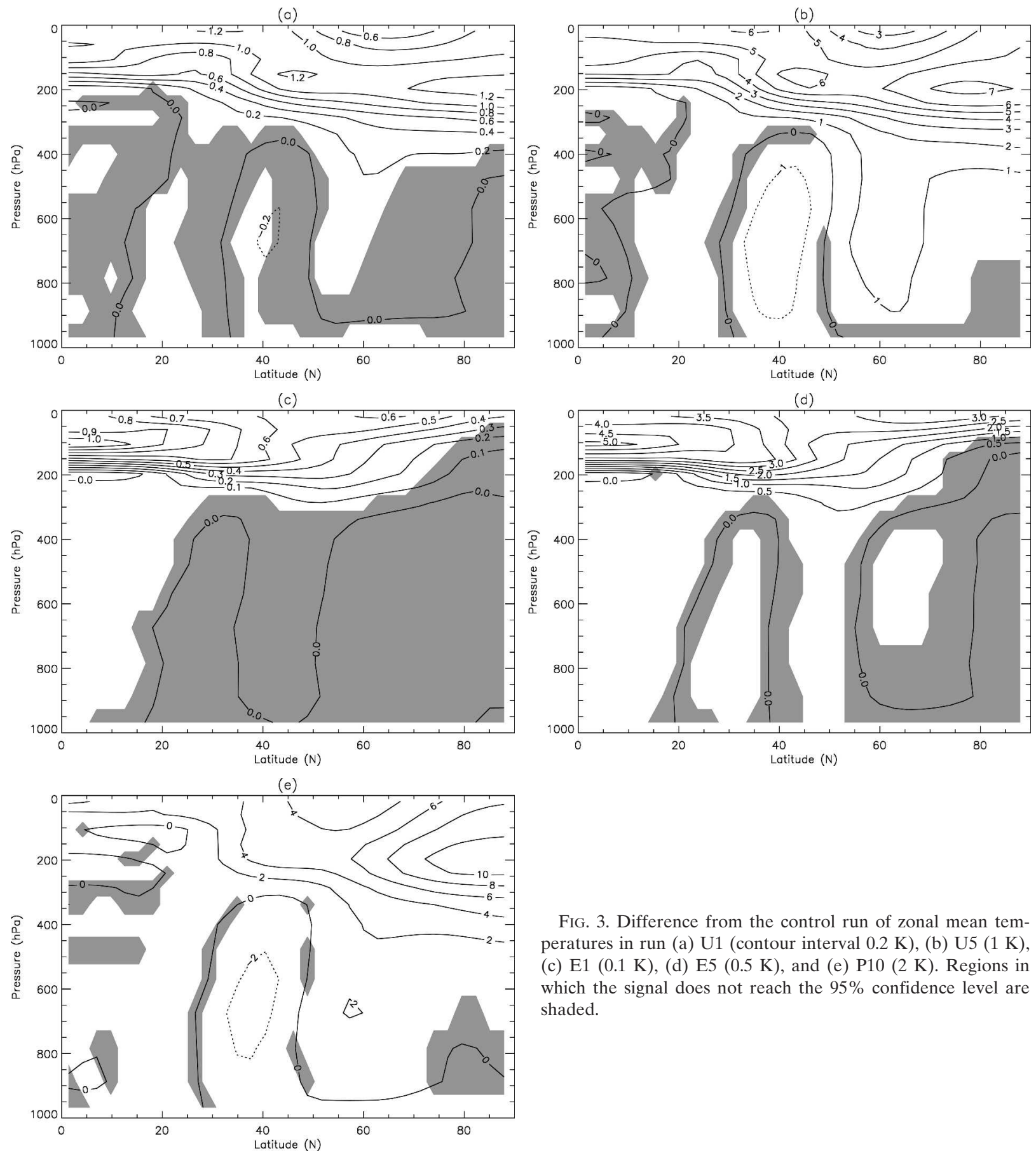

FIG. 3. Difference from the control run of zonal mean temperatures in run (a) U1 (contour interval $0.2 \mathrm{~K}$ ), (b) U5 (1 K), (c) E1 $(0.1 \mathrm{~K}),(\mathrm{d}) \mathrm{E} 5(0.5 \mathrm{~K})$, and (e) P10 (2 K). Regions in which the signal does not reach the $95 \%$ confidence level are shaded.

For experiments P10, U5, U1, and E5 there is a statistically unambiguous response throughout the troposphere and stratosphere. For E1, the signal in the stratosphere and low-latitude troposphere has a $95 \%$ confidence level; for the rest of the troposphere the confidence interval of the results is about $80 \%$. However, the almost identical patterns shown by the E5 and E1 runs suggest that, in these regions, the response is real. The contour intervals in the plots have been chosen so that the value of the 5-K-amplitude experiments is a factor 5 larger than that for the respective $1-\mathrm{K}$ experiments; from this it is clear that the response is approximately linear in the stratosphere, but that in the troposphere the 5-K experiments show a slightly larger than linear response (factor of 6-7, rather than a factor of 5). 

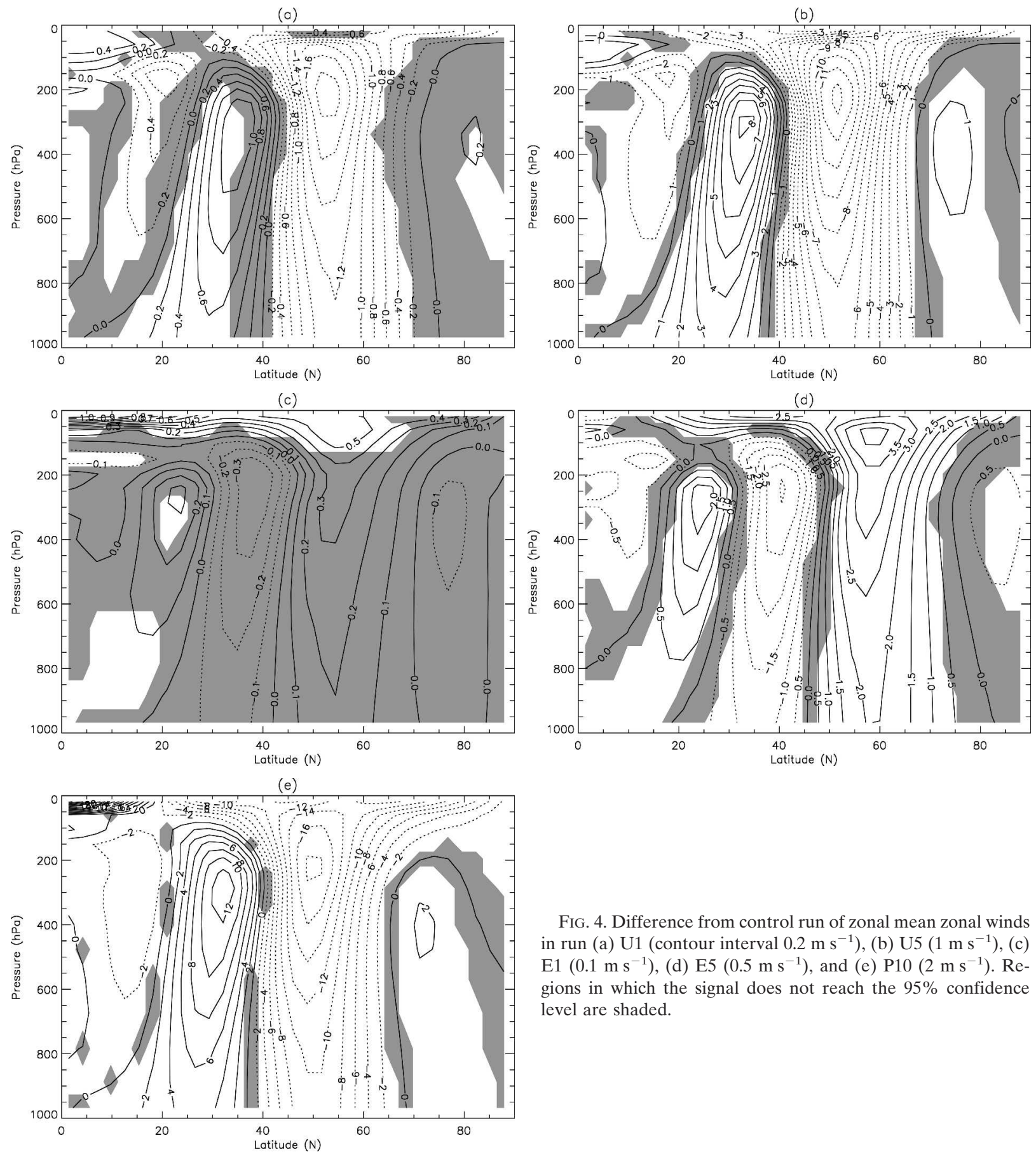

FIG. 4. Difference from control run of zonal mean zonal winds in run (a) U1 (contour interval $0.2 \mathrm{~m} \mathrm{~s}^{-1}$ ), (b) U5 $\left(1 \mathrm{~m} \mathrm{~s}^{-1}\right)$, (c) E1 $\left(0.1 \mathrm{~m} \mathrm{~s}^{-1}\right)$, (d) E5 $\left(0.5 \mathrm{~m} \mathrm{~s}^{-1}\right)$, and (e) P10 $\left(2 \mathrm{~m} \mathrm{~s}^{-1}\right)$. Regions in which the signal does not reach the $95 \%$ confidence level are shaded.

The pattern of response in zonal mean temperatures in the $\mathrm{E}$ runs is similar to that reported as resulting from increased solar activity in both NCEP-NCAR data (Haigh 2003) and full GCM (fGCM) studies (Haigh 1996, 1999; Larkin et al. 2000). The sGCM runs can, in no way, be considered as simulations of solar effects, so a direct comparison of the results is not appropriate, but the similarity of the resulting patterns may allow us to draw conclusions as to a potential mechanism for solar influence on tropospheric climate.

\section{2) ZONAL WIND}

The perturbations in the zonal mean zonal wind found in the sGCM experiments are presented in Fig. 4. As would be anticipated, from thermal wind consider- 
ations of the temperature perturbations discussed above, banded structures appear in the troposphere in each case.

In runs U1 and U5 (Figs. 4a and 4b), these appear as a band of weaker winds near $55^{\circ}$ and a band of stronger winds near $35^{\circ}$. This represents a weakening of the jet and an equatorward shift. There are some interesting qualitative similarities between the results of the $\mathrm{U}$ experiments and those shown for the response to stratospheric aerosol in Fig. 1e in the NCEP-NCAR zonal winds.

The response of the E experiments (Fig. 4d) is again somewhat different from the $\mathrm{U}$ runs, with a negative band near $40^{\circ}$ flanked by positive bands near $25^{\circ}$ and $60^{\circ}$, respectively. These correspond to a weakening and latitudinal expansion, but mainly a poleward shift, of the jets. Now the pattern is more qualitatively similar to the solar signal found in the NCEP-NCAR data (Fig. 1b).

Experiment P10 (Fig. 4e) produces a similar pattern of response to U5 in the midlatitudes, but with larger magnitudes. This represents a significant equatorward shift of the jets, although with no real weakening; the results of P10 also differ from U5 in that the response in the tropical lower stratosphere shows a large westerly acceleration in the former and very little effect in the latter. Experiment P10 is very similar (apart from a change in sign of the forcing) to that of Polvani and Kushner (2002), who presented results of the response of a similar model to polar stratospheric cooling and presented an equivalent poleward shift of the jets.

Clearly, the responses seen in the simple model experiments U5 (or P10) and E5 are much larger than those that are found to be the result of volcanic or solar influences in the NCEP-NCAR data analysis. Experiments U1 and E1, using forcings that were a factor of 5 smaller than U5 and E5, respectively, might be more appropriate for interpretation in this regard. However, because the patterns of response seen in the $1-\mathrm{K}$ experiments are essentially identical to those of the corresponding 5-K runs, it seems clear that the same processes are operating. On this basis, and for reasons of clarity and brevity, our analysis and interpretation of the data, below, is based on the 5-K experiments alone.

\section{3) EDDY FLUXES AND FEEDBACKS}

Because the sGCM results are in qualitative agreement with both the NCEP-NCAR anomalies for solar and volcanic forcing and the fGCM results, we seek to understand the mechanisms whereby forcing in the lower stratosphere leads to the modeled responses throughout the troposphere. We now ask to what ex-
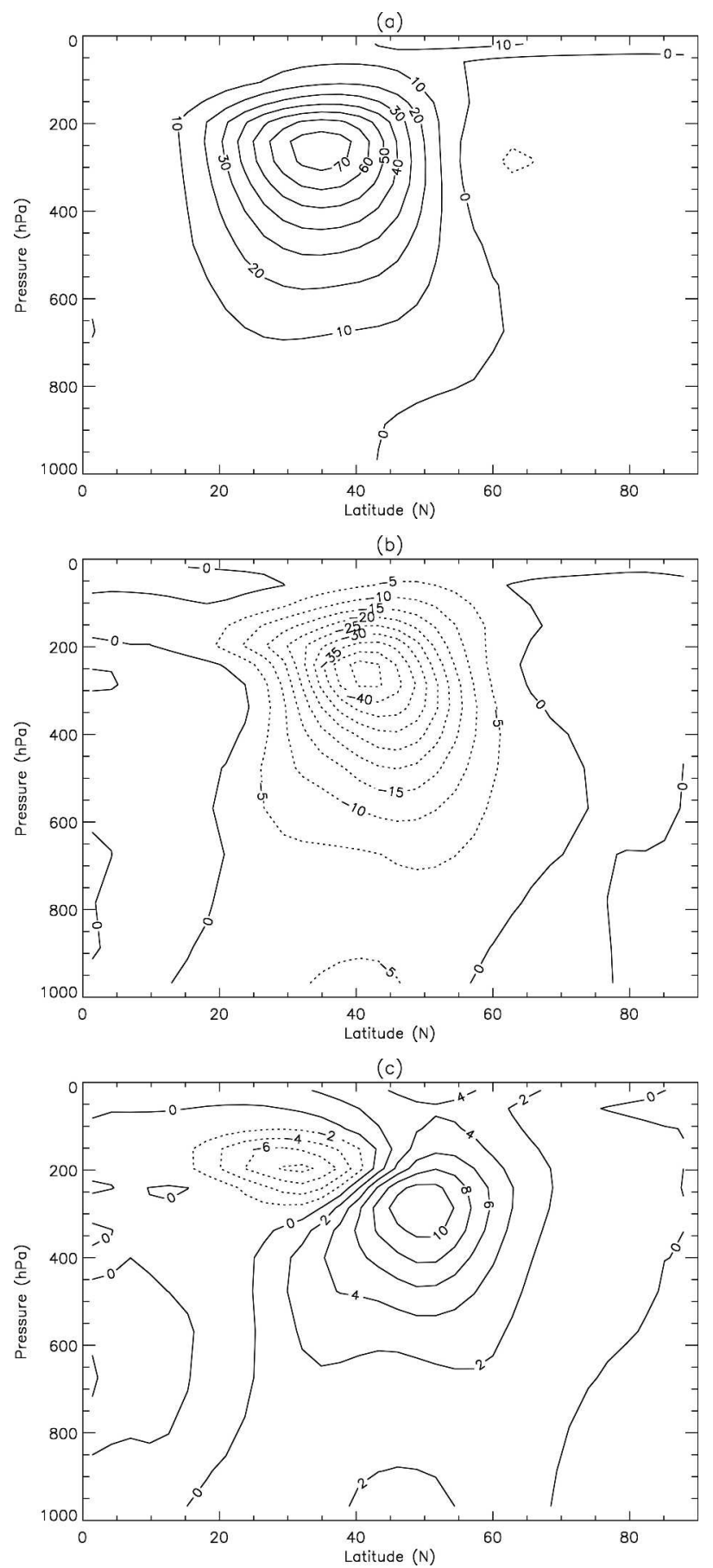

FIG. 5. Poleward flux of westerly momentum by the zonally asymmetric eddies $\left[u^{\prime} v^{\prime}\right]\left(\mathrm{m}^{2} \mathrm{~s}^{-2}\right)$ for (a) the control run $\mathrm{C}$, (b) the U5-C anomaly, and (c) the E5-C anomaly.

tent these mechanisms are zonally symmetric or involve feedbacks from the (transient) eddies.

The fields of poleward eddy momentum flux and poleward eddy heat flux are shown for control run $\mathrm{C}$ in Figs. 5a and $6 a$, respectively. The anomalies in these 


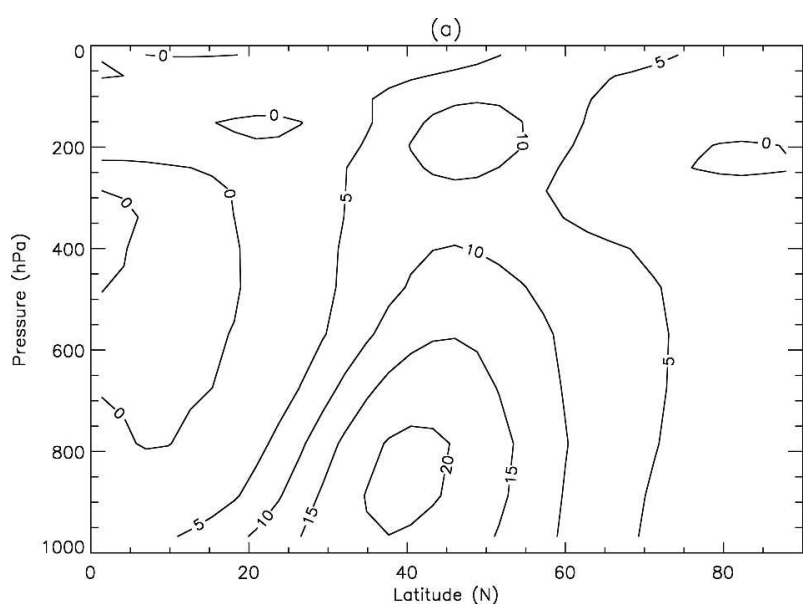

(b)

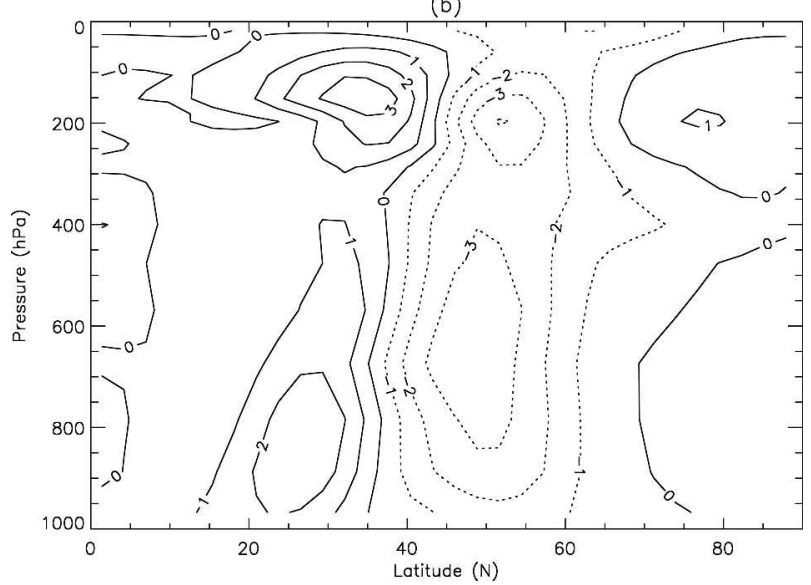

(c)

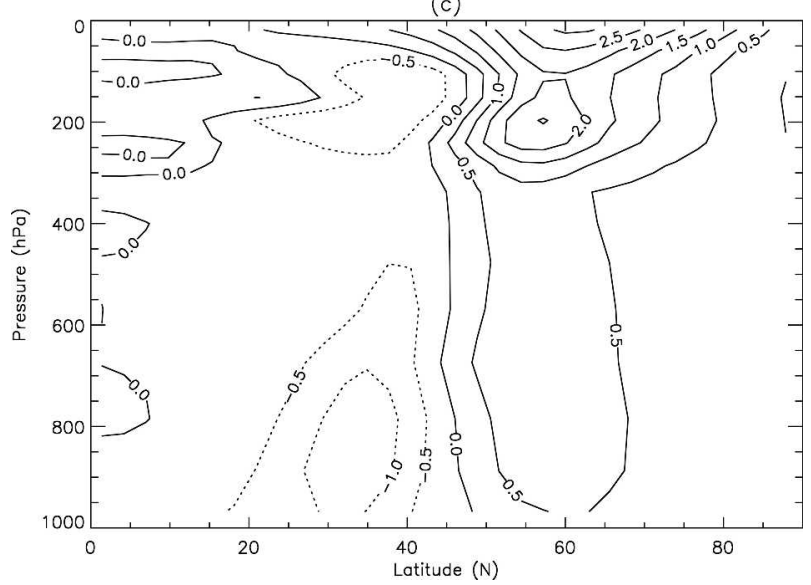

FIG. 6. Poleward temperature flux by the zonally asymmetric eddies $\left[v^{\prime} T^{\prime}\right]\left(\mathrm{K} \mathrm{m} \mathrm{s}^{-1}\right)$ for (a) the control run $\mathrm{C}$, (b) the U5-C anomaly, and (c) the E5-C anomaly.

fields, relative to run $\mathrm{C}$, for runs U5 and E5 are shown in the other panels of the same figures. For run U5 the momentum flux is significantly weakened, particularly on the poleward side of the midlatitude jet. The latitudinal gradient of the $\left[u^{\prime} v^{\prime}\right]$ anomaly implies anomalous easterly forcing by the eddies poleward of the peak anomaly at $45^{\circ}$, and westerly forcing equatorward of the peak. Comparison with the zonal wind anomalies in Fig. 4a reveals that these forcing regions coincide with wind anomalies of the same sign. The eddy forcing peaks in the upper troposphere, driving the zonal mean state away from thermal wind balance there. Balance is maintained by an anomalous zonally symmetric overturning circulation, whose Coriolis acceleration balances the eddy forcing locally in the upper troposphere, which yields a low-level anomaly balance between the drag and a Coriolis acceleration of the opposite sign to that in the upper troposphere. The streamfunction of the anomalous meridional circulation for run U5 (Fig. $7 b)$ coincides with the anomalous eddy forcing and zonal wind in precisely this manner.

The anomalous meridional circulation also acts on the zonal temperature state, by vertical advection of potential temperature in its vertical branches in such a way as to maintain thermal wind balance in the presence of the eddy momentum forcing. This acts to increase the meridional temperature gradient below the upper-level westerly eddy forcing and reduce it below easterly eddy forcing. Comparison of the anomalous meridional circulation in Fig. $7 \mathrm{~b}$ and the temperature anomaly in Fig. 3a for run U5 reveals a coincidence between the anomalous mean descent and the warm anomaly poleward of $45^{\circ}$, and between the anomalous mean ascent and the cold anomaly equatorward of $45^{\circ}$. Finally, to complete the picture, thermal forcing by the anomalous meridional circulation is balanced locally by a combination of anomalous convergence of the northward eddy heat flux (Fig. 6b) and diabatic heating. The former comprises an equatorward shift and weakening of the poleward heat flux. The latter is inevitable in the sGCM experiments, in which all thermal anomalies in the troposphere are linearly damped by the Newtonian relaxation, because the tropospheric reference state is unchanged in the integrations.

A similar relationship between the various anomaly fields holds for experiment E5, although the anomalies are of a smaller magnitude. The northward eddy momentum flux anomaly (Fig. 5c) now has a dipolar structure, comprising a strengthening of the flux on the poleward flank of the jet and a weakening on its equatorward flank. This corresponds to a tripolar pattern of anomalous momentum flux convergence that coincides with the zonal wind anomalies in Fig. 4b. The lowlatitude change is larger, however, so that the net effect is to weaken the jet and displace it poleward. The anomalous meridional circulation (Fig. 7c) locally counteracts the anomalous momentum forcing, while its vertical branches coincide with the temperature 


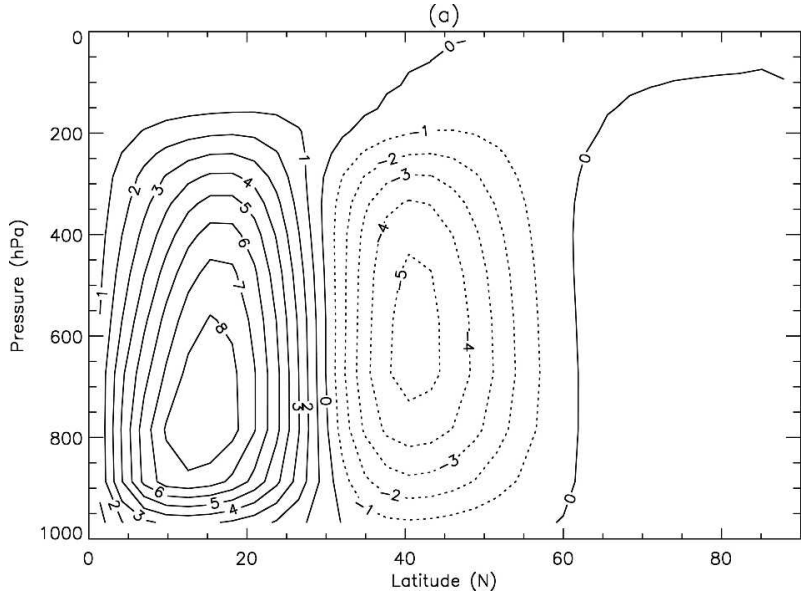

(b)

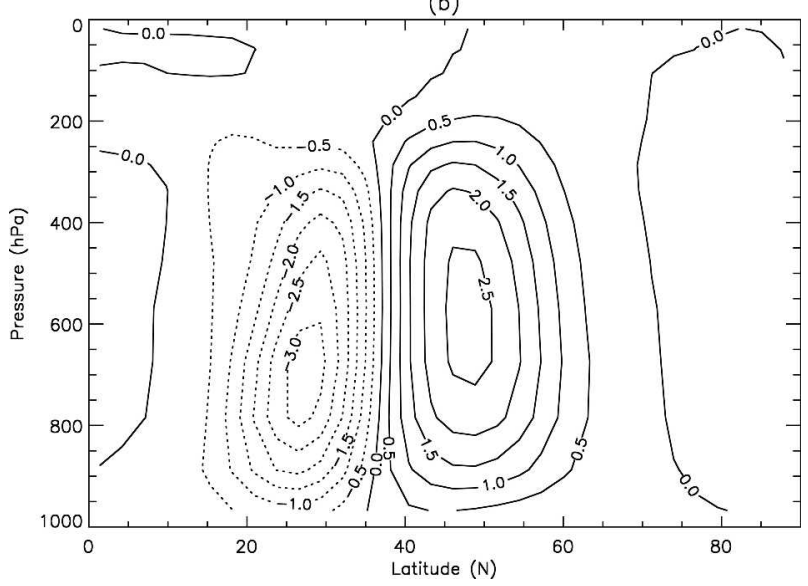

(c)

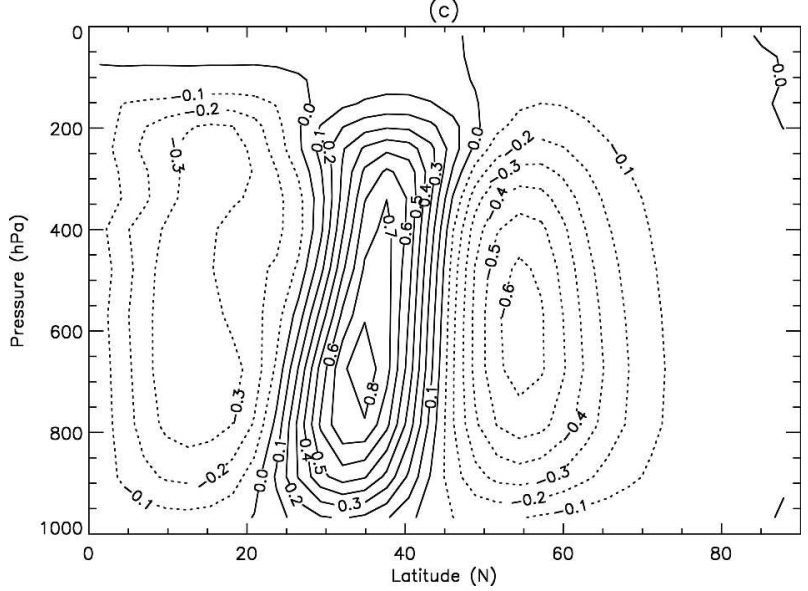

FIG. 7. Streamfunction of the mean meridional circulation $\left(10^{10} \mathrm{~kg} \mathrm{~s}^{-1}\right)$ for (a) the control run C, (b) the U5-C anomaly, and (c) the E5-C anomaly. The streamfunction is defined in the appendix.

anomalies in Fig. 3b. The anomalous northward eddy heat fluxes act to damp the temperature anomalies and balance the effects of the anomalous meridional circulation.

In both U5 and E5 we obtain a consistent picture, comprising coincident changes in the strength and location of the midlatitude jet and its associated storm track. The anomalous momentum flux is up gradient, because it acts to maintain the zonal wind anomaly, whereas the anomalous heat flux is down gradient, which is coincident with the anomalous meridional temperature gradient.

Transformed Eulerian mean (TEM) diagnostics of the sGCM experiments provide a description that is equivalent to the Eulerian diagnostics presented above, but with no additional insight into reasons for the meridional displacements of the midlatitude jets in the anomaly experiments. In run U5, the Eliassen-Palm (E-P) fluxes (not shown) are weaker and displaced equatorward, with significantly less equatorward wave propagation into the subtropics. The associated anomalous E-P flux convergence represents weaker easterly forcing by the eddies at upper levels, particularly in the subtropics. However, the latitudinal distribution of the anomalous eddy forcing does not match that of the anomalous zonal mean wind, and there is a large local cancellation between eddy momentum forcing and Coriolis acceleration that is associated with the TEM meridional circulation, as noted by Hoskins (1983), just as in the Eulerian diagnostic framework. Similar arguments apply to run E5, although the pattern of TEM anomalies differs from that of run U5. Now the E-P flux is shifted poleward relative to the control and does not propagate as high, particularly on the equatorward flank of the jet, where the tropopause has been lowered (Fig. 3b).

\section{4) Vertically integRated MOMENTUm BUDGETS}

The vertically integrated budget of westerly momentum provides a useful constraint in interpreting the sGCM perturbation experiments. Because the model's low-level flow is damped by the linear drag parameterization, latitudinal displacements in the westerly jet must be maintained by anomalous poleward momentum fluxes, which are up gradient in the zonal wind anomalies.

Terms in the vertically integrated momentum budget for the control experiment, shown in Fig. 8a, confirm that the surface stress is accurately balanced at each latitude by the convergence of the poleward flux of westerly momentum [uv], and that this is dominated by its eddy component $\left[u^{\prime} v^{\prime}\right]$. Both the midlatitude surface westerlies and the easterly trade winds are maintained by the poleward momentum flux. The divergence of the mean circulation term $[u][v]$ is generally small, and reflects the latitudinal structure of the upper-tropospheric branches of the Hadley and Ferrel cells. It opposes the 

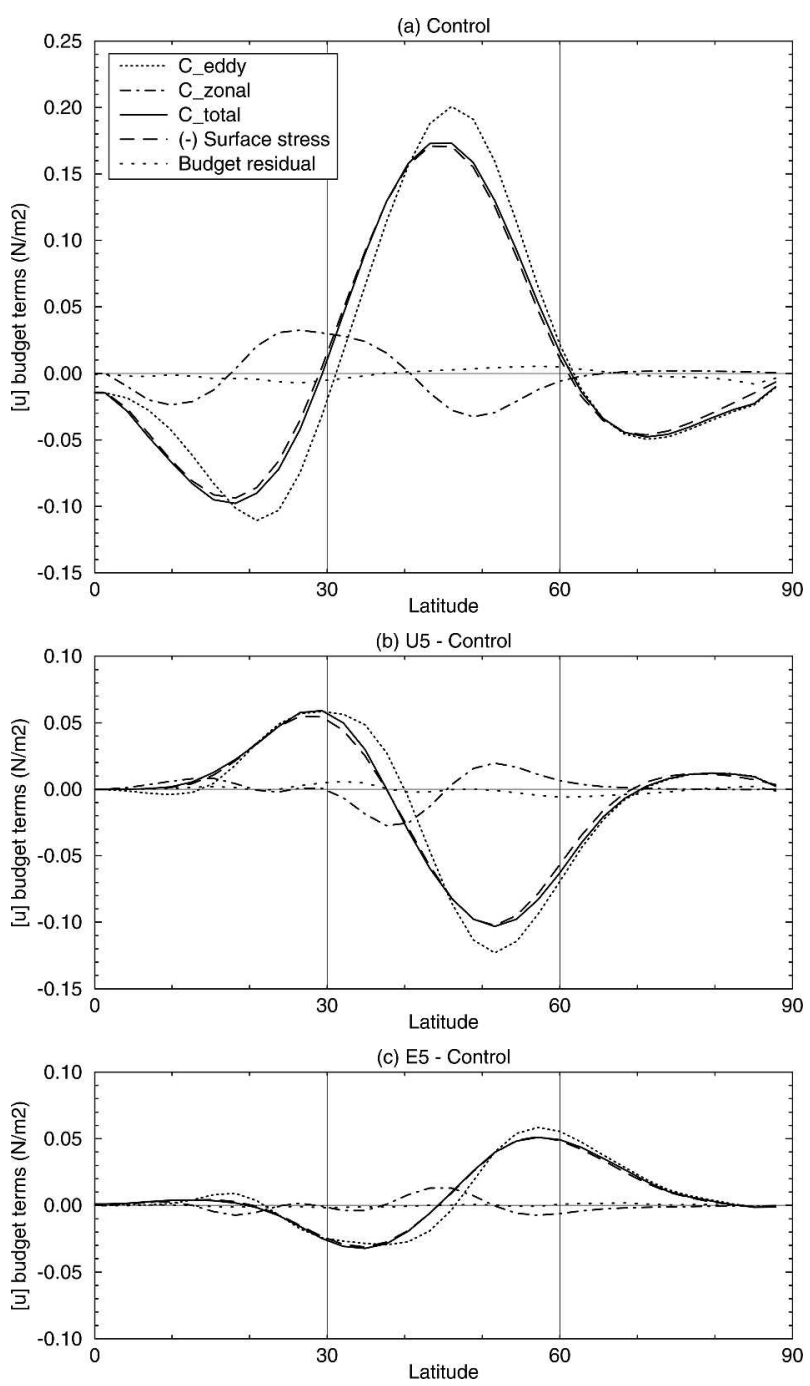

FIG. 8. Terms in the vertically integrated budget of the westerly momentum [see Eq. (A1) in the appendix] for (a) the control run C, (b) the U5-C anomaly, and (c) the E5-C anomaly. Each panel shows the convergence of the flux of the westerly momentum resulting from the zonally asymmetric eddies $\left(C_{\text {EDDY }}\right.$, dotted $)$, the zonally averaged flow $\left(C_{\text {ZONAL }}\right.$, dash-dot $)$, and their sum ( $C_{\text {TOTAL }}$, solid $)$; the surface stress $\left(\left[\tau_{S \lambda}\right]\right.$, dashed $)$; and the budget residual (faint dotted). All quantities are units of $\mathrm{Nm}^{-2}$.

eddy flux convergence in midlatitudes and leads to a small displacement of the eddy flux convergence and surface stress in the subtropics.

The momentum budget anomalies in experiments U5 and E5, relative to the control, are shown in Figs. 8b and $8 \mathrm{c}$. It is clear that the eddy flux convergence dominates the anomalies, balancing the surface stress dipole associated with the equatorward jet shift in U5 and the tripole associated with the poleward shift in E5. The mean circulation term is small in comparison, but accounts for a small displacement between the peak stress anomaly and peak eddy flux convergence anomaly on the equatorward side of the jet in each experiment.

We conclude that the jet displacements in the sGCM experiments are primarily maintained by the changes in the poleward eddy momentum flux. The anomalous eddy flux convergence is concentrated in the upper troposphere, driving an anomalous mean circulation (displacement of the Ferrel cell), which acts to transport the anomalous momentum downward and, so, balance the surface stress anomaly.

\section{c. Interpretation}

We now ask to what extent the diagnostic picture presented in the preceding section can be interpreted causally, beyond the statement that the imposed stratospheric changes lead to the modeled changes in the troposphere. All of the experiments involve long-termaveraged steady states, in which the anomalous mean flow and eddy forcing terms in the budget equations must balance. The vertically integrated momentum balance immediately implies that latitudinal displacements of the jets necessarily involve up-gradient anomalous momentum fluxes, which we have found to be dominated by the eddy component, while the thermal wind balance implies that the baroclinic component of such displacements will be associated with anomalous temperature gradients.

In a study of the extratropical response to tropical forcing that is associated with ENSO, Seager et al. (2003) observed a similar relationship to that found here between anomalous eddy forcing and perturbations of the zonal mean state. They used the upgradient nature of the anomalous momentum fluxes and the down-gradient nature of the anomalous heat fluxes to imply a causality chain, starting with changes to the subtropical upper troposphere that altered the eddy momentum fluxes, with these leading to the heat flux changes. This interpretation was reinforced by a diagnosis of wave propagation in a linear wave model, suggesting that ENSO-driven perturbations of the zonal mean state in the subtropics lead to the changes that are observed in the meridional wave propagation and, therefore, in the eddy momentum fluxes.

Our sGCM results are consistent with such an interpretation, beginning with changes in the thermal structure of the tropopause region that are associated with the modified stratospheric reference state. However, other sGCM experiments, in which the tropospheric baroclinicity was altered (not shown), exhibit the same relationship between the eddy and mean flow perturbations. Thus, the up- or down-gradient nature of the different eddy fluxes is not, in itself, a sufficient indication of a detailed causality chain. Moreover, the sGCM 
control run (and, indeed, the atmosphere itself) comprises an extratropical jet stream and associated storm track, which exhibits the same qualitative balance, with the eddies driven by baroclinic instability. The heat flux is down gradient and the momentum fluxes are up gradient, driving a thermally indirect meridional circulation (Ferrel cell) in the Eulerian mean, which is necessary to maintain the thermal wind balance in the presence of the eddy forcing. The sGCM stratospheric forcing experiments necessarily involve perturbations to and latitudinal displacements of this basic balance.

What we can conclude from the sGCM experiments is that imposed changes in the lower-stratospheric temperature forcing lead to coherent changes in the latitudinal location and width of the midlatitude jet stream and its associated storm track, and that eddy/mean flow feedbacks are crucial to these changes. Imposed stratospheric warming and an associated lowering of the tropopause tends to weaken the jet and storm-track eddies; equatorial stratospheric warming displaces the jet poleward, while uniform or polar warming displaces it markedly equatorward.

We intend to perform further sGCM experiments to investigate the more detailed causality chain, including ensemble spinup experiments and the use of a zonally symmetric version of the sGCM, in which eddy forcing is prescribed. Kushner and Polvani (2004) have recently used a similar methodology in an investigation of the high-latitude stratosphere-troposphere interaction that is associated with annular variability.

\section{Conclusions}

A multiple regression analysis of the NCEP-NCAR reanalysis zonal mean zonal wind data reveals a pattern of response to solar variability in which the subtropical jets weaken and move poleward during periods of higher solar activity, with the effect transmitted throughout the vertical extent of the troposphere. This pattern is similar in both shape and magnitude to that previously estimated to be the result of the sun's 11-yr cycle in UV radiation using global circulation models. The response in the data to volcanic aerosol is found to be a weakening and equatorward shift of the jets.

The experiments with the simplified global circulation model provide some indications as to how these responses arise. All runs in which thermal perturbations were applied only in the lower stratosphere show effects throughout the troposphere, with the vertically banded anomalies in the temperature and zonal wind, and changes in the tropospheric mean circulation, which is typical of the results of data analysis. Heating the lower stratosphere increases the static stability in this region, lowers the tropopause, and reduces the wave fluxes here. This leads to coherent changes through the depth of the troposphere, involving the location and width of the jet stream, storm-track, and eddy-induced meridional circulation.

The precise shape of the patterns of response depend on the distribution of the stratospheric heating perturbation: heating at mid- to high latitudes causes the jets to move equatorward and the Hadley cells to shrink, while heating only at the low latitudes results in a poleward shift of the jets and an expansion of the Hadley cells. Low-latitude heating also results in a weakening of the subtropical jets. The results of the low-/highlatitude heating experiments, thus, appear to be qualitatively similar to the responses found in the observational data to higher solar activity/volcanic aerosol; and the low-latitude heating experiment is similar to the global circulation model simulations of the effects of enhanced solar UV.

The success of the simplified global circulation model in reproducing qualitatively the observed tropospheric response to imposed stratospheric perturbations also suggests that moist feedbacks are not a crucial component of the response; it is eddy/mean flow feedbacks that are the primary mechanism. It is also interesting to note that, despite the presence of a uniform stratosphere, the lack of a stratospheric polar vortex, and the use of broad latitudinal-scale perturbations, the sGCM is able to reproduce the tropospheric patterns. This suggests that a detailed representation of the stratosphere is not necessary for understanding the tropospheric aspects of solar influence, although the source of the stratospheric heating remains an important factor.

We, therefore, suggest that the observed climate response to solar variability is brought about by a dynamical response in the troposphere to heating predominantly in the stratosphere. The effect is small, and frequently masked by other factors, but is not negligible in the context of the detection and attribution of climate change. The results also suggest that, at the earth's surface, the climatic effects of solar variability will be most easily detected in the subtropics and midlatitudes.

Acknowledgments. The data analysis was carried out by $\mathrm{RD}$ as part of an undergraduate vacation work placement at Imperial College. The modeling work was funded by the European Community SOLICE project and by the U.K. Natural Environment Research Council. We are grateful to Myles Allen for the regression program, to Brian Hoskins for helpful discussion, and to two anonymous referees whose comments led to improvements in the paper. 


\section{APPENDIX}

\section{Circulation Diagnostics}

\section{a. Mean meridional circulation}

The zonally averaged divergent circulation may be visualized by a mass streamfunction $\psi$ in the meridional plane, defined by

$$
[v]=\frac{g}{c} \frac{\partial[\psi]}{\partial p}, \quad[\omega]=-\frac{g}{c} \frac{\partial[\psi]}{a \partial \phi}, \quad c=2 \pi a \cos \phi,
$$

where [] denotes a zonal average, $v$ is the northward velocity, and $\omega=(D p / D t)$ is the vertical motion in isobaric coordinates. The factor $(g / c)$ produces a mass streamfunction on the sphere in kilograms per second. In practice, $\psi$ is computed by integrating the zonally averaged northward wind downward from the top of atmosphere in isobaric coordinates, independently at each latitude:

$$
[\psi]=\int[v] d M=\frac{c}{g} \int_{0}^{p}[v] d p^{\prime} .
$$

\section{b. Vertically integrated westerly momentum budget}

The zonally averaged balance of westerly momentum in isobaric, and spherical coordinates can be written as

$$
\begin{aligned}
\frac{\partial[u]}{\partial t}= & -\frac{1}{a \cos ^{2} \phi} \frac{\partial}{\partial \phi}\left\{[u v] \cos ^{2} \phi\right\}-\frac{\partial}{\partial p}[u \omega]+f[v] \\
& +\left[F_{\lambda}\right],
\end{aligned}
$$

where $F_{\lambda}$ is the zonal friction force, and the other symbols have their usual meanings. Integrating over the depth of the atmosphere and taking a long-time average, the Coriolis term vanishes by mass continuity, while small terms involving the surface pressure variations and the surface vertical momentum flux that are associated with the resolved flow can be neglected (as has been confirmed by the model results). The resulting balance of vertically integrated westerly momentum becomes

$$
\begin{aligned}
\frac{\partial}{\partial t} \int_{0}^{p_{s}}[u] d p= & -\frac{1}{a \cos ^{2} \phi} \frac{\partial}{\partial \phi}\left\{\cos ^{2} \phi \int_{0}^{p_{s}}([u][v]\right. \\
& \left.\left.+\left[u^{\prime} v^{\prime}\right]\right) d p\right\}+\int_{0}^{p_{s}}\left[F_{\lambda}\right] d p,
\end{aligned}
$$

or

$$
\frac{1}{g} \frac{\partial}{\partial t} \int_{0}^{p_{s}}[u] d p=C_{\mathrm{ZONAL}}+C_{\mathrm{EDDY}}-\left[\tau_{S \lambda}\right]
$$

Here, the momentum flux convergence has been partitioned into contributions from the zonally averaged and zonally asymmetric flows, $[u][v]$ and $\left[u^{\prime} v^{\prime}\right]$, respectively, where ()$^{\prime}$ denotes a local departure from the zonal average. Following Held and Suarez (1994), the sGCM incorporates Rayleigh friction in the lower atmosphere with an inverse time scale

$$
k_{v}=k_{f} \max \left\{0,\left(\sigma-\sigma_{b}\right) /\left(1-\sigma_{b}\right)\right\},
$$

where $\sigma_{b}=0.7$, and the surface value is $k_{f}=1 \mathrm{day}^{-1}$. The resulting zonal friction force $F_{\lambda}=-k_{v} u$ in Eq. (A1), integrates to give the zonally averaged zonal component of the surface stress

$$
\left[\tau_{S \lambda}\right]=\frac{1}{g} \int_{0}^{p_{s}} k_{v}[u] d p,
$$

which is computed as a discrete sum in the model coordinates.

\section{REFERENCES}

Gleisner, H., and P. Thejll, 2003: Patterns of tropospheric response to solar variability. Geophys. Res. Lett., 30, 1711, doi:10.1029/2003GL017129.

Gray, L. J., S. J. Phipps, T. J. Dunkerton, M. P. Baldwin, E. F. Drysdale, and M. R. Allen, 2001: The influence of the equatorial upper stratosphere on Northern Hemisphere stratospheric sudden warmings. Quart. J. Roy. Meteor. Soc., 127, 1985-2003.

Haigh, J. D., 1994: The role of stratospheric ozone in modulating the solar radiative forcing of climate. Nature, 370, 544-546. , 1996: The impact of solar variability on climate. Science, $\mathbf{2 7 2}$, 981-984.

— 1999: A GCM study of climate change in response to the 11-year solar cycle. Quart. J. Roy. Meteor. Soc., 125, 871-892. , 2003: The effects of solar variability on the Earth's climate. Philos. Trans. Roy. Soc. London, 361A, 95-111.

Hansen, J., M. Sato, and R. Ruedy, 1997: Radiative forcing and climate response. J. Geophys. Res., 102, 6831-6864.

Held, I. M., and M. J. Suarez, 1994: A proposal for the intercomparison of the dynamical cores of atmospheric general circulation models. Bull. Amer. Meteor. Soc., 75, 1825-1830.

Hoskins, B. J., 1983: Modeling of the transient eddies and their feedbacks on the mean flow. Large-Scale Dynamical Processes in the Atmosphere, B. J. Hoskins and R. P. Pearce, Eds., Academic Press, 169-199.

— the semi-implicit method. Quart. J. Roy. Meteor. Soc., 101, 637-655.

Kodera, K., and Y. Kuroda, 2002: Dynamical response to the solar cycle. J. Geophys. Res., 107, 4749, doi:10.1029/2002JD002224.

Kushner, P. J., and L. M. Polvani, 2004: Stratosphere-troposphere coupling in a relatively simple AGCM: The role of eddies. $J$. Climate, 17, 629-639.

Larkin, A., J. D. Haigh, and S. Djavidnia, 2000: The effect of solar UV irradiance variations on the Earth's atmosphere. Space Sci. Rev., 94, 199-214. 
Polvani, L. M., and P. J. Kushner, 2002: Tropospheric response to stratospheric perturbations in a relatively simple general circulation model. Geophys. Res. Lett., 29, 1114, doi:10.1029/ $2001 \mathrm{GL} 014284$.

Randel, W. J., F. Wu, and D. J. Gaffen, 2000: Interannual variability of the tropical tropopause derived from radiosonde data and NCEP reanalyses. J. Geophys. Res., 105, 15509 15524.

Sato, M., J. Hansen, M. P. McCormick, and J. B. Pollack, 1993: Stratospheric aerosol optical depths, 1850-1990. J. Geophys. Res., 98, 22 987-22 994.

Seager, R., N. Harnik, Y. Kushnir, W. Robinson, and J. Miller, 2003: Mechanisms of hemispherically symmetric climate variability. J. Climate, 16, 2960-2978.
Shindell, D. T., G. A. Schmidt, M. E. Mann, D. Rind, and A. Waple, 2001: Solar forcing of regional climate change during the Maunder Minimum. Science, 294, 2149-2152.

Simmons, A. J., and D. M. Burridge, 1981: An energy and angular-momentum conserving vertical finite-difference scheme and hybrid vertical coordinates. Mon. Wea. Rev., 109, 758766.

van Loon, H., and D. Shea, 2000: The global 11-year signal in July-August. Geophys. Res. Lett., 27, 2965-2968.

von Storch, H., and F. W. Zwiers, 1999: Statistical Analysis in Climate Research. Cambridge University Press, 484 pp.

Wuebbles, D. J., C. F. Wei, and K. O. Patten, 1998: Effect on stratospheric ozone and temperature during the Maunder Minimum. Geophys. Res. Lett., 25, 523-526. 